\title{
Human Adipose Stem Cell-Conditioned Medium Increases Survival of Friedreich's Ataxia Cells Submitted to Oxidative Stress
}

\author{
Jonathan Jones, Alicia Estirado, Carolina Redondo, Carlos Bueno, and Salvador Martínez
}

Friedreich's ataxia (FA) is a multisystemic disorder characterized by progressive gait, ataxia, and cardiomyopathy. There are few treatments for this disease; thus, we analyzed in vitro the possible beneficial effect of adult stem cells in FA. To this end, human adipose stem cells from healthy individuals and periodontal ligament cells from FA patients were isolated and cultured. FA cells are especially vulnerable to oxidative stress; thus, they were submitted to this condition and cultured in adipose stem cell-conditioned medium. This resulted in increased cell survival and upregulation of oxidative-stress-related genes as well as frataxin, among other genes. A number of trophic factors were shown to be expressed by the adipose stem cells, especially brain-derived neurotrophic factor (BDNF), which was also identified in the conditioned medium. The culture of the ataxic cells under oxidative stress and in the presence of this trophic factor confirmed its protective effect. Thus, this work demonstrates that adipose stem cell-conditioned medium from healthy individuals is capable of changing the transcription levels of oxidative-stress-related genes in cells that are particularly susceptible to this condition, avoiding cellular degeneration. Also, this work shows how neurotrophic factors, particularly BDNF, are capable of increasing cell survival in response to oxidative stress, which occurs in many neurodegenerative diseases.

\section{Highlights}

1. Periodontal ligament cells from Friedreich's ataxia (FA) patients are susceptible to oxidative stress.

2. Adipose-tissue-derived stem cell-conditioned medium reduces active caspase- 3 levels and increases cell survival.

3. Adipose stem cell-conditioned medium contains a number of trophic factors, brain-derived neurotrophic factor (BDNF) being the most significant.

4. BDNF is a vital trophic factor to protect FA cells from oxidative stress.

\section{Introduction}

TAXIC DISORDERS are a group of rare diseases character-
ized by the progressive loss of motor functions and co-
ordination, generally resulting in the premature death of the
patient. There are numerous types of ataxia, Friedreich's
ataxia (FA) being the most prevalent. This disorder is caused
by a GAA-triplet repeat expansion in the frataxin gene, re-
sulting in an overall loss of frataxin protein, located in the
mitochondria [1]. As frataxin is involved in iron homeostasis,
its malfunction provokes an accumulation of iron in the cells,
causing its death [2]. The cells that are most sensitive to this phenomenon are neurons (especially large sensory neurons of the dorsal root ganglia in the spinal cord), heart (causing cardiomyopathy), and the pancreas (many FA patients are also diabetic) [3,4]. The main cause of death of the patients is by heart failure, and there is currently no cure for the disease, while the few treatments that are currently in use do not ameliorate the progressive neurodegeneration.

Stem cells have been proven to be a potent tool for the treatment of many diseases, including neurodegenerative disorders. It has been shown in various animal studies that stem cells are capable of stopping degenerative processes or even regenerate lost tissue. This occurs due to several mechanisms: cell fusion [5-8], trophic factor release [9-11], immunomodulation [12], transdifferentiation $[13,14]$, or activation of host progenitor cells $[15,16]$.

In our lab, we have previously shown that bone marrow stem cells are capable of protecting degenerating neurons in demyelinating, motorneuron degenerating, and cerebellar ataxia animal models [14,17-19, respectively]. Previous works have proven that adipose stem cells have similar effects as bone marrow [20]. Thus, we decided to investigate the use of adult stem cells in a FA model. Specifically, we will analyze the possible neuroprotective effect stem cells may have on degenerating ataxic cells. To this end, we isolated and expanded periodontal ligament cells from FA

Neuroscience Institute UMH-CSIC, University Miguel Hernández, San Juan, Spain. 
patients, in order to study in vitro the beneficial effects adult stem cells may exert. Since FA cells are sensitive to oxidative stress, we submitted the cells to this condition and cocultured the cells in adipose stem cell-derived conditioned medium. The results of this work show how stem-cellmediated trophic factor release may increase cell survival under oxidative stress conditions.

\section{Experimental Procedures}

\section{Adipose-tissue-derived stem cell isolation and culture}

Human adipose tissue was obtained by liposuction from healthy individuals, who previously signed a written consent, and immediately refrigerated. No more than $12 \mathrm{~h}$ after the extraction, the adipose tissue was processed. First, the tissue was washed extensively in phosphate-buffered saline (PBS; Invitrogen) supplemented with 1\% penicillin/streptomycin (Invitrogen). Afterward, it was digested in $0.2 \mathrm{mg} / \mathrm{mL}$ animal-component-free collagenase-I (Worthington) for $45 \mathrm{~min}$ at $37^{\circ} \mathrm{C}$, followed by mechanical dissociation. Then, the tissue was washed extensively to remove the collagenase and centrifuged at $1,500 \mathrm{rpm}$ for $10 \mathrm{~min}$. The resulting pellet was washed and centrifuged twice, and counted in a Neubauer chamber before placing in culture. The culture medium used was animal-origin-free mesenchymal stem cell medium (StemCells Technologies), and the cells were placed in a cell culture flask at a concentration of $1 \times 10^{6}$ cells / flask. The medium was changed $48 \mathrm{~h}$ after the initial plating and afterward every 3-4 days. After 7-10 days, when the culture was almost confluent, the cells were detached using TrypLE Select animal-origin-free stable trypsin replacement (Gibco) and replated at a concentration of 50,000 cells/flask. For the experiments, cells from passages 2-3 were used, which considered to be adipose-derived stem cells (Fig. 1A). To confirm this, immunocytochemical staining for CD34, CD44, CD90, and CD45 was performed (see Fig. 1A-D).

\section{Periodontal-ligament-derived stem cell isolation and culture}

Before extraction, the patients were informed of the procedures to be performed and signed a written consent. The teeth used were deciduous teeth in the process of falling out. The teeth, either after spontaneously falling or by extraction in a dental clinic, were immediately taken into the laboratory and processed. After extracting the periodontal ligament, the tissue was processed in a similar fashion as the adipose tissue (collagenase treatment, followed by mechanical dissociation and culture). The cells used were at passages $3-5$. In the case where periodontal ligament cells from healthy individuals were used, this was taken from the biobank of our institution. This cell population is a mixture of different individuals so as to maintain patient's privacy. The procedure to extract the cells was the same; only in this case the teeth were taken from individuals of various ages.

\section{Immunocytochemical analysis}

A standard immunocytochemical protocol was used. First, the cells were fixed in $4 \%$ paraformaldehyde, washed, and blocked with $10 \%$ goat serum, $5 \%$ bovine albumin, $0.025 \%$ triton, and PBS. Afterward, they were incubated overnight at room temperature with the primary antibody, diluted in blocking solution ( $10 \%$ goat serum, $5 \%$ bovine albumin, and PBS). The following primary antibodies were used: mouse antiCD44 (1:200; BD Pharmingen), mouse anti-CD45 (1:200; AbD Serotec), mouse anti-CD34 (1:200; BD Pharmingen), mouse anti-CD90 (1:200; BD Pharmingen), and rabbit anti-cleaved caspase-3 (1:400; Cell Signaling Technology). The following day, the cells were incubated with the secondary antibodies, which were either Alexa Fluor antibodies (1:500; Molecular Probes) or biotinylated secondary antibodies (1:200; Vector Laboratories) followed by an incubation with streptavidin conjugated with Cy3 (1:500). DAPI (Molecular Probes) or Hoechst (Sigma-Aldrich) was used to stain nuclei. Histological samples were observed and micrographs were taken under a fluorescence microscope (Leica DMR; Leica Microsystems).

\section{Hydrogen peroxide, brain-derived neurotrophic factor, and TrkB blocking treatment of cultured cells}

Adipose stem cells from healthy individuals and periodontal ligament cells from healthy and FA patients were cultured in standard medium and supplemented with $0.1 \mathrm{mM}$ hydrogen peroxide (Sigma-Aldrich) for $48 \mathrm{~h}$. Hydrogen peroxide was added twice, once at the beginning of the experiment and $24 \mathrm{~h}$ afterward. To obtain the adipose stem cell-conditioned media, this was recovered $24 \mathrm{~h}$ after incubation under normal culture conditions. Also, conditioned medium from adipose stem cells exposed to hydrogen peroxide for $24 \mathrm{~h}$ was recovered. Both conditioned media were centrifuged at $1,500 \mathrm{rpm}$ for $10 \mathrm{~min}$ and the resulting pellet was discarded. Also, periodontal ligament cells were cultured in the same hydrogen peroxide medium [labeled $\mathrm{L}(\mathrm{H})$ in the Figs. 3 and 4]. Depending on the stem-cellconditioned media, 2 different experimental conditions were performed: periodontal ligament cells cultured in $0.1 \mathrm{mM}$ hydrogen peroxide and in adipose stem cell-conditioned medium $[\mathrm{L}(\mathrm{H})+\mathrm{A}$ in the Figs. 3 and 4], and periodontal ligament cells exposed to hydrogen peroxide and in conditioned medium from adipose stem cells that had previously been submitted to oxidative stress $[\mathrm{L}+\mathrm{A}(\mathrm{H})]$.

For the cultures where brain-derived neurotrophic factor (BDNF) was added, this trophic factor was included along with the hydrogen peroxide for 2 days, at several concentrations (1-100 ng/mL; Sigma Aldrich), while in the cell cultures with the TrkB blocker (recombinant human TrkB FC Chimera; R\&D Systems), $100 \mathrm{ng} / \mathrm{mL}$ was used.

\section{Real-time quantitative PCR}

Total mRNA of the cells was isolated using the Trizol protocol (Invitrogen). Five micrograms of mRNA was reverse-transcribed, and $100 \mathrm{ng}$ of cDNA was amplified by real-time PCR using Power SYBR Green Master mix (Applied Biosystems). All the samples were run in triplicate using the StepOne Plus Real-Time PCR system (Applied Biosystems) and analyzed with the StepOne Software. Analyses were carried out using the delta $\mathrm{C}(\mathrm{T})$ method and calculated relative to glyceraldehyde 3-phosphate dehydrogenase (GAPDH) (forward: AGCCACATCGCTCAGACAC, reverse: GCCCAATACGACCAAATCC). The following primers were used, taken from the PrimerBank webpage 
(http://pga.mgh.harvard.edu/primerbank/): frataxin (forward: AAGACTAGCAGAGGAAACGCT, reverse: ACCCAG TTTGACAGTTAAGACAC), superoxide dismutase 1 (SOD1) (forward: AGGGCATCATCAATTTCGAGC, reverse: GCCCA CCGTGTTTTCTGGA), superoxide dismutase 2 (SOD2) (forward: AACCTCAGCCCTAACGGTG, reverse: AGCAGCAA TTTGTAAGTGTCCC), transferrin receptor (forward: CCCT CGTGAGGCTGGATCT, reverse: CTATACGCCACATAACC CCCA), H-ferritin (forward: TCCTACGTTTACCTGTCCA TGT, reverse: GTTTGTGCAGTTCCAGTAGTGA), L-Ferritin (forward: ACGAGCGTCTCCTGAAGATG, reverse: CCCAG GGCATGAAGATCCAAA), BDNF (forward: TAACGGCGG CAGACAAAAAGA, reverse: TGCACTTGGTCTCGTAGAA GTAT), glial-derived neurotrophic factor (GDNF) (forward: GGCAGTGCTTCCTAGAAGAGA, reverse: AAGACACAAC CCCGGTTTTTG), neurotrophin-3 (NT3) (forward: AGACT CGCTCAATTCCCTCAT, reverse: GCAGTTCGGTGTCCAT TGC), neurotrophin-4 (NT4) (forward: CTGTGTGCGATGC AGTCAGT, reverse: TGCAGCGGGTTTCAAAGAAGT), vascular endothelial growth factor (VEGF) (forward: CGCAGC TACTGCCATCCAAT, reverse: GTGAGGTTTGATCCGCAT AATCT), platelet-derived growth factor-A (forward: GCAAG ACCAGGACGGTCATTT, reverse: GGCACTTGACACTGCT CGT), basic fibroblast growth factor (forward: AGTGTGTG CTAACCGTTACCT, reverse: ACTGCCCAGTTCGTTTCA GTG), insulin-like growth factor 1 (forward: GGAGCTGTG ATCTAAGGAGGC, reverse: GGGCTGATACTTCTGGGT CTT), nerve growth factor (NGF) (forward: TGTGGGTTGG GGATAAGACCA, reverse: GCTGTCAACGGGATTTGGGT), epidermal growth factor (forward: TGGATGTGCTTGATAA GCGG, reverse: ACCATGTCCTTTCCAGTGTGT), caspase-3 (forward: CATGGAAGCGAATCAATGGACT, reverse: CTG TACCAGACCGAGATGTCA), NADPH oxidase 1 (NOX1, forward: TTGTTTGGTTAGGGCTGAATGT, reverse: GCCAA TGTTGACCCAAGGATTTT), NOX2 (forward: CCATCCGG AGGTCTTACTTTGA, reverse: ACGTACAATTCGTTCAGC TCCA), NOX3 (forward: CGTGGCGCATTTCTTCAACC, reverse: GCTCTCGTTAGGGGTGTTGC), NOX4 (forward: TTG GGGCTAGGATTGTGTCTA, reverse: GAGTGTTCGGCACA
TGGGTA), NOX5 (forward: ATTGGCTGGGTACACGGTTC, reverse: CGAGGAGGTAGGACAGGTGA), Dual oxidase 1 (DUOX1, forward: GCAGCGATTTGATGGGTGGTA, reverse: AGGTGGGGTTCTCCCAAGG), DUOX2 (forward: CTGGGTCCATCGGGCAATC, reverse: GTCGGCGTAATT GGCTGGTA), glutathione peroxidase 1 (GPX-1) (forward: CAGTCGGTGTATGCCTTCTCG, reverse: GAGGGACGCC ACATTCTCG), Catalase (forward: TGGGATCTCGTTGGA AATAACAC, reverse: TCAGGACGTAGGCTCCAGAAG), superoxide dismutase 3 (SOD3) (forward: ATGCTGGCGC TACTGTGTTC, reverse: ACTCCGCCGAGTCAGAGTT).

\section{Western blot analysis}

The lysis buffer and process used were similar to that of Palomo et al. [21]. Cell lysates and culture media were separated on $15 \%$ sodium dodecyl sulfate-polyacrylamide gels, and probed for mouse anti-frataxin (1:750; Santa Cruz Biotechnology) and rabbit anti-BDNF (1:500; Santa Cruz Biotechnology), respectively. Secondary antibodies were visualized by chemiluminescence (ECL; Amersham). For protein quantification we used Quantity One software (BioRad).

\section{Statistical analysis}

Statistical significance between control and experimental groups was calculated with Sigmaplot v11.0 software, using the paired $t$-test.

\section{Results}

\section{Adipose stem cells exposed to hydrogen peroxide increase the expression levels of genes implicated in oxidative stress and iron metabolism}

Adipose-tissue-derived stem cells were isolated, cultured, and characterized as seen in Figure 1. These cells, as in bone marrow mesenchymal stem cells, express the surface markers CD44 and CD90, and do not express CD34 and CD45
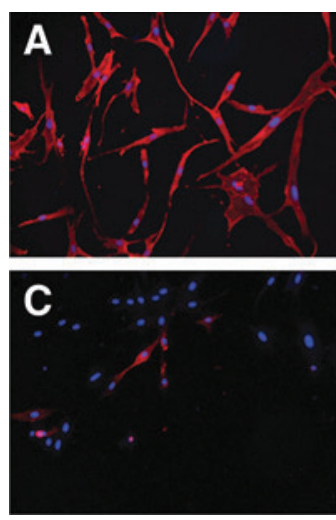
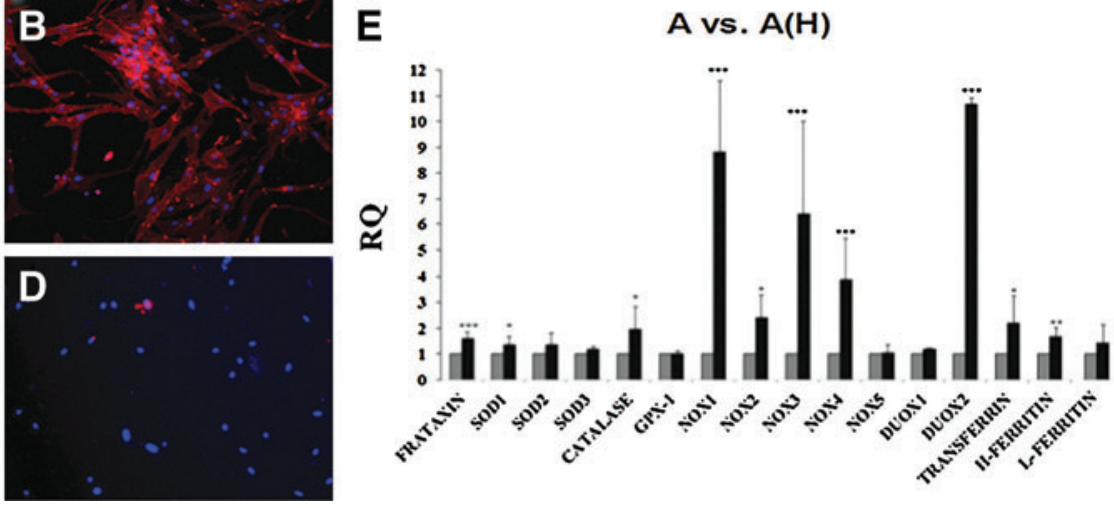

FIG. 1. Characterization and effect of oxidative stress in adipose-derived stem cells of healthy individuals. (A-D) Surface markers of adipose-derived stem cells. These stem cells express many markers similar to bone marrow mesenchymal stem cells, including CD90 (A) and CD44 (B), and do not express CD34 (C) or CD45 (D). In all images, red is the antibody indicated, and blue is DAPI staining. (E) Real-time PCR analysis of several genes comparing adipose stem cells under normal culture conditions (A, gray bar) to the cells cultured in the presence of hydrogen peroxide [A(H), black bar]. Adipose stem cells under normal conditions is considered as control (value $=1)$. Y-axis indicates the relative quantity of the genes analyzed $(R Q)$. All genes are expressed relative to GAPDH. Error bars indicate average deviations: ${ }^{*} P<0.05$, ${ }^{* *} P<0.005$, and ${ }^{* * *} P<0.001$ $(n=6)$. Color images available online at www.liebertpub.com/scd 
(Fig. 1A-D). Also, periodontal ligament cells from FA patients were isolated and cultured. These cells express several stem cell and neural markers, due to their neural crest origin [22].

The effect of hydrogen peroxide on the expression of several genes in adipose-tissue-derived stem cells of healthy individuals was analyzed (Fig. 1E). The genes studied were superoxide dismutases (SOD1, SOD2, and SOD3), hydrogen peroxide neutralizing genes (Catalase and GPX-1), NADPH oxidases (NOX1, NOX2, NOX3, NOX4, and NOX5), dual oxidases (DUOX1 and DUOX2), as well as genes implicated in iron metabolism (frataxin, transferrin, H-ferritin, and L-ferritin). As it can be seen in the histogram, the adipose stem cells exposed to hydrogen peroxide expressed increased transcription levels of several oxidative-stress-related genes, including SOD1, catalase, and several of the NOX and DUOX2. Also, almost all the iron-related genes, except for $L$ ferritin, were upregulated. As the cause for the degeneration in FA is due to low levels of frataxin and subsequent iron accumulation, the possible upregulation of these genes in FA patients is of utmost importance. Overall, these results indicate a possible compensatory response of the cells in order to increase cell survival due to the oxidative stress conditions, increasing overall the metabolism of the cell.

\section{Comparative gene expression between periodontal ligament cells isolated from healthy and FA individuals}

Periodontal ligament cells were extracted from both healthy and FA individuals, and the basal expression levels of the genes commented in the previous section were analyzed under normal culture conditions (Fig. 2A). As a result, FA cells expressed significantly lower levels of frataxin (see Fig. 2B for western blot analysis), as well as the majority of the genes analyzed. On the other hand, 2 genes, NOX3 and DUOX1, presented higher levels of expression. Previous studies in yeast and human cells have shown that antioxidant enzymes are affected due to the frataxin defect, and are not capable of responding to oxidative stress conditions, corroborating our results $[23,24$, respectively].

\section{Adipose-tissue-derived stem cell-conditioned medium increases cell survival and upregulates the expression levels of genes implicated in oxidative stress and iron metabolism, in cultured cells isolated from FA patients}

The periodontal ligament cells from FA patients were submitted to oxidative stress, as in the case of the adipose stem cells. Cell death was calculated both using Hoechst (Fig. 3A-D) and active caspase-3 staining (Fig. 3E-H). The following culture conditions were performed: periodontal ligament cells under normal culture conditions (Fig. 3A), periodontal ligament cells exposed to hydrogen peroxide (Fig. $3 \mathrm{~B})$, periodontal ligament cells exposed to hydrogen peroxide and in adipose stem cell-conditioned medium (Fig. 3C), and periodontal ligament cells exposed to hydrogen peroxide and in conditioned medium from adipose stem cells previously submitted to oxidative stress (Fig. 3D). In the case of Hoechst staining, $20.71 \% \pm 4.56 \%$ of periodontal ligament cells exposed to hydrogen peroxide presented pyknotic nuclei indicating that the cells were undergoing cell death processes (Fig. 3E), as opposed to $1.76 \% \pm 0.74 \%$ and $0.44 \% \pm 0.58 \%$ of the periodontal ligament cells cultured under 1 of the 2 adipose stem cellconditioned medium (Fig. 3F, G; $n=3$ ). As for active caspase-3 staining, $9.55 \% \pm 2.55 \%$ of the periodontal ligament cells exposed to hydrogen peroxide expressed active caspase-3 (Fig. 3E). Meanwhile, in the cultures with adipose stem cell-conditioned medium (Fig. 3F-G), only $1.40 \% \pm 0.38 \%$ (in the case of FA periodontal ligament cells under stem-cell-conditioned medium) and $1.69 \% \pm 0.25 \%$ (in the case of FA cells under conditioned medium from stem cells that have been previously submitted to oxidative stress) were active caspase- 3 positive $(n=3)$.

Active caspase- 3 was also analyzed by quantitative PCR (Fig. $3 \mathrm{H})$, corroborating the immunocytochemistry results, indicating that stem-cell-conditioned medium increases the cell survival of FA cells exposed to hydrogen peroxide. In the
A

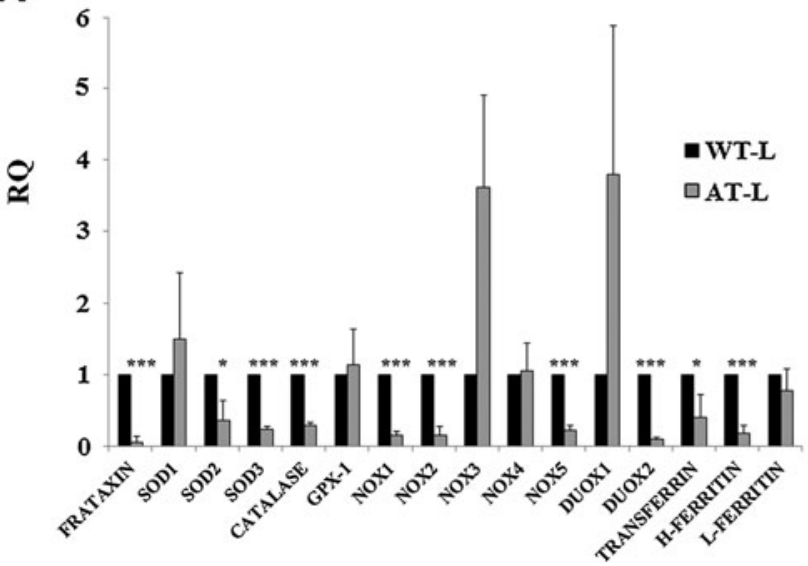

B

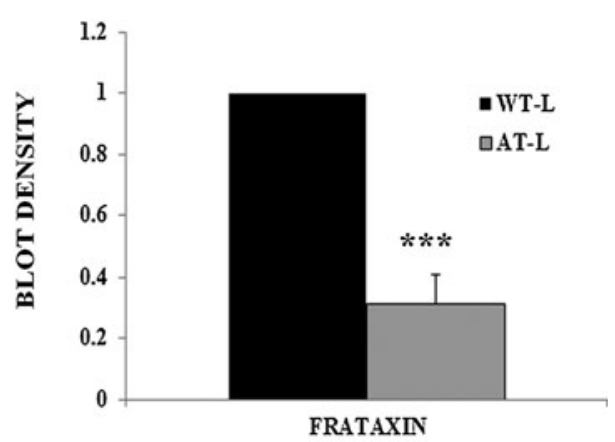

FIG. 2. Comparative analysis of periodontal ligament cells isolated from healthy and Friedreich's ataxia (FA) patients under normal culture conditions. (A) Analysis of periodontal ligament cells of healthy (WT-L, black bars) and FA individuals (AT-L, gray bars). The cells from healthy individuals are considered as control (value $=1$ ). Y-axis indicates the relative quantity of the genes analyzed (RQ). ${ }^{*} P<0.05$ and ${ }^{* * *} P<0.005$. (B) Frataxin expression, as detected by western blot analysis, and quantifying band density (Y-axis) of healthy (WT-L) and FA (AT-L) individuals $(P<0.001, n=4)$. 

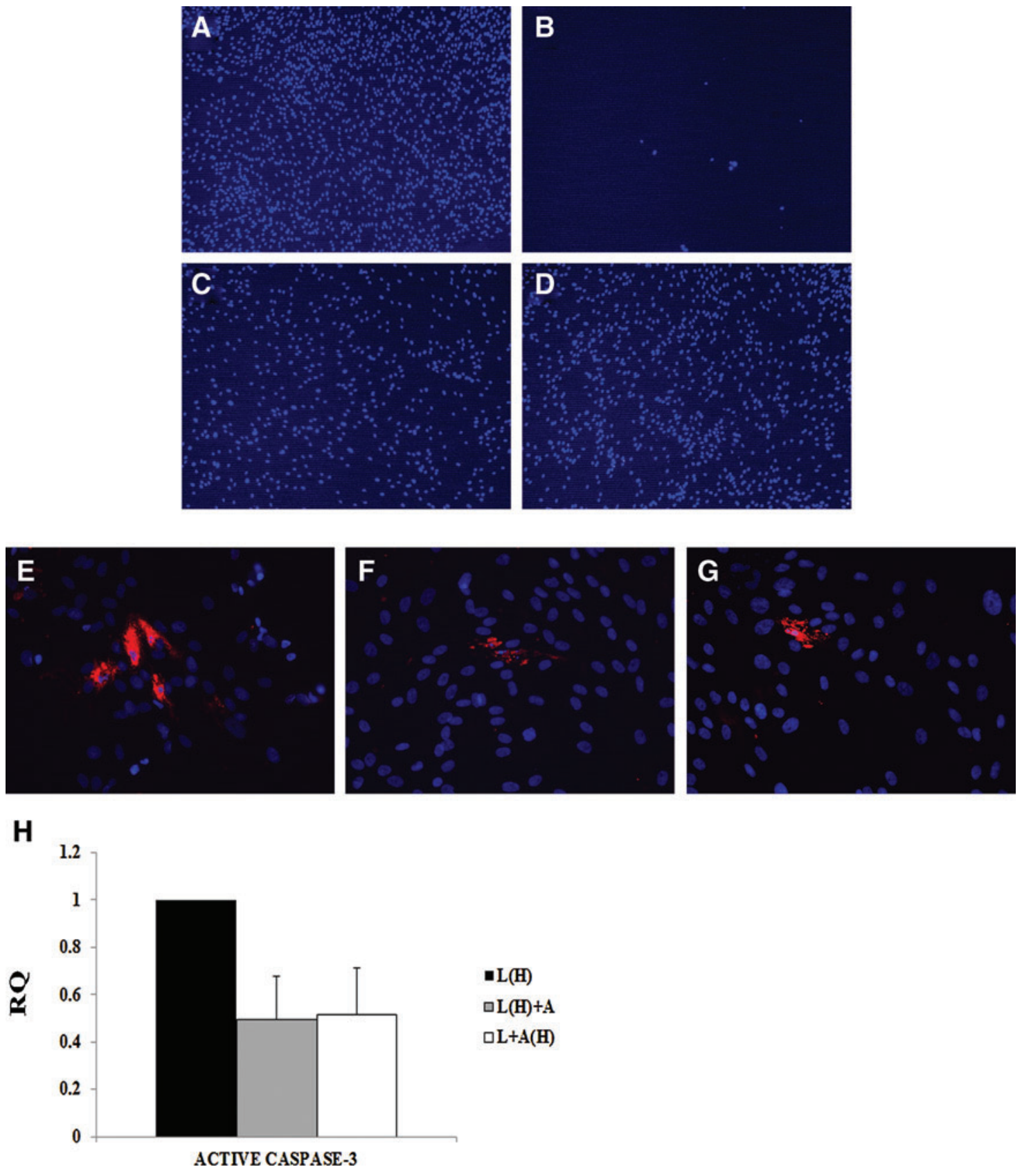

FIG. 3. Caspase-3 is activated in response to oxidative stress. (A-D) Hoechst staining of periodontal ligament cells in the various culture conditions: with standard culture medium (A), exposed to hydrogen peroxide (B), exposed to hydrogen peroxide and in adipose stem cell-conditioned medium (C), and exposed to hydrogen peroxide and in conditioned medium from adipose stem cells previously submitted to oxidative stress (D). Images taken at $40 \times$ magnification. (E-G) Active caspase-3 staining (red) of periodontal ligament cells exposed to hydrogen peroxide (E), exposed to hydrogen peroxide and in adipose stem cell-conditioned medium (F), and exposed to hydrogen peroxide and in conditioned medium from adipose stem cells previously submitted to oxidative stress (G). Y-axis indicates the relative quantity of the genes analyzed (RQ). Nuclei are stained with Hoechst. All images were taken at $200 \times$ magnification. (H) QPCR of active caspase-3. $n=3$ in all cases. Color images available online at www.liebertpub.com/scd

Fig. $3 \mathrm{H}, \mathrm{L}(\mathrm{H})+\mathrm{A}$ indicates periodontal ligament cells exposed to hydrogen peroxide and in adipose stem cell-conditioned medium, whereas $\mathrm{L}+\mathrm{A}(\mathrm{H})$ is periodontal ligament cells exposed to hydrogen peroxide and under conditioned medium from adipose stem cells previously exposed to oxidative stress. Also, $\mathrm{L}(\mathrm{H})$ indicates periodontal ligament cells exposed to hydrogen peroxide.
Also, quantitative expression of oxidative stress and ironmetabolism-related genes were analyzed and compared in periodontal ligament cells under normal culture conditions and in the presence of hydrogen peroxide (Fig. 4A). As a result, it was observed that the expression levels of the genes analyzed in periodontal ligament cells from FA patients under oxidative stress either did not change or were even 
A

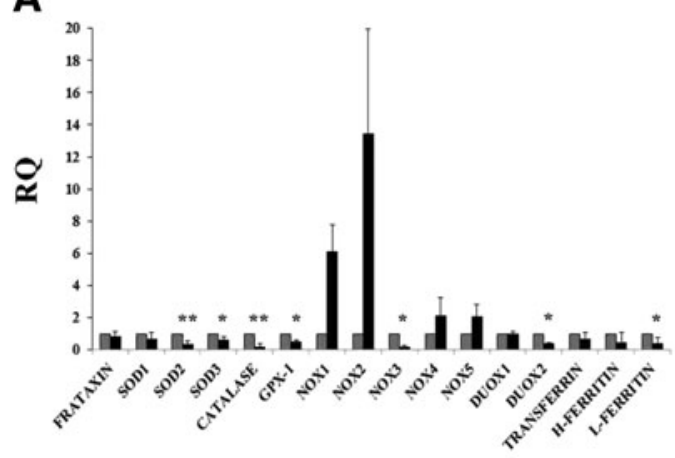

C

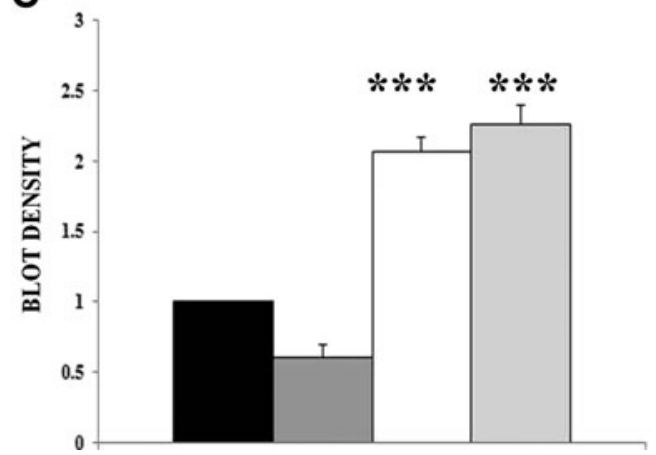

B

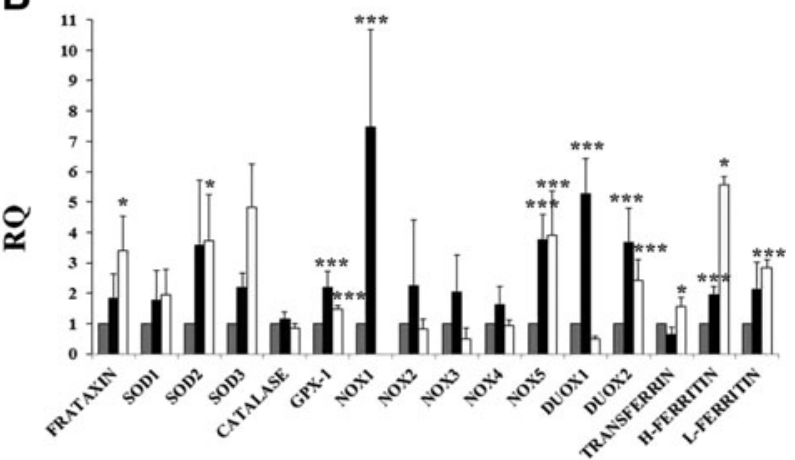

D

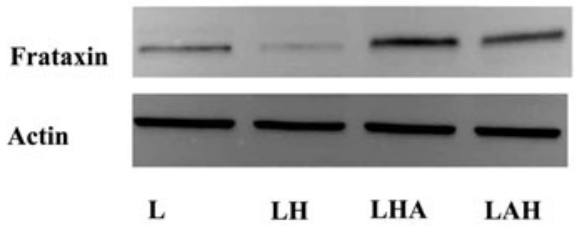

FIG. 4. Gene and protein expression in response to oxidative stress in FA cells cultured with adipose-tissue-derived stem cell-conditioned medium. (A) Gene expression in periodontal ligament cells under normal culture media (labeled L in the histogram, gray bars), which is considered as control, and compared with cells exposed to hydrogen peroxide [L(H), black bars]. Error bars indicate average deviations: ${ }^{*} P<0.05$, ${ }^{* *} P<0.005$, and ${ }^{* * *} P<0.001(n=6)$. (B) Transcription comparison analysis in periodontal ligament cells exposed to hydrogen peroxide [L(H), taken as control, gray bars], exposed to hydrogen peroxide and in adipose stem cell-conditioned medium [ $\mathrm{L}(\mathrm{H})+\mathrm{A}$, black bars], and exposed to hydrogen peroxide and in conditioned medium from adipose stem cells previously submitted to oxidative stress $[\mathrm{L}+\mathrm{A}(\mathrm{H})$, white bars]. NOX1 was not detected in $\mathrm{L}+\mathrm{A}(\mathrm{H})$. In (A) and (B), Y-axis indicates the relative quantity of the genes analyzed (RQ). All genes are expressed relative to GAPDH. Error bars indicate average deviations: ${ }^{*} P<0.05,{ }^{* *} P<0.005$, and ${ }^{* * *} P<0.001$. (C, D) Western blot analysis of frataxin in periodontal ligaments under standard culture medium (taken as control, black bar), exposed to hydrogen peroxide (dark gray bar), exposed to hydrogen peroxide and in adipose stem cell-conditioned medium (white bar), and exposed to hydrogen peroxide and in conditioned medium from adipose stem cells previously submitted to oxidative stress (light gray bar) ( $n=6$ in control and experimental groups, $n=3$ in western blot analysis).

decreased. Only NOX1 and NOX2 were upregulated. This was opposed to what was observed in the adipose stem cells from healthy individuals (see Fig. 1E, almost all the genes were upregulated). However, when the periodontal ligament cells exposed to hydrogen peroxide were cultured under stem-cell-conditioned medium, there was an overall upregulation of the genes analyzed (Fig. 4B). Of the 2 adipose stem cell-conditioned media used, the one taken from stem cells that had previously been exposed to hydrogen peroxide $[\mathrm{L}+\mathrm{A}(\mathrm{H})$ in the Fig. $4 \mathrm{C}]$ presented the highest amount of frataxin upregulation, increasing its expression $\sim 4$ times. Also, SOD2, GPX-1, NOX5, DUOX2, transferrin, H-ferritin, and $L$-ferritin were increased. Increased frataxin expression at the protein level was corroborated by western blot analysis (Fig. 4C, D).

\section{Adipose-tissue-derived stem cells secrete several trophic factors in response to hydrogen peroxide}

As the adipose stem cell-conditioned medium, especially from cells that had previously been under oxidative stress conditions, was sufficient to increase oxidative-stress-related genes as well as frataxin, we decided to analyze the conditioned medium for trophic factors that may induce this upregulation. A total of 10 trophic factors were analyzed by quantitative PCR in the adipose stem cells (Fig. 5A). The result was that there was a significant increase in BDNF, VEGF, and NGF when comparing adipose stem cell-conditioned medium exposed to hydrogen peroxide $[\mathrm{A}(\mathrm{H})$ in the figure] compared with standard culture conditions (A in the Fig. 5A). Specifically, BDNF was the trophic factor that increased the most, with a 5-fold increase, compared with VEGF and NGF, which presented a more modest increase $(<2$-fold).

Further, western blot analysis demonstrated that BDNF was secreted in higher quantities in the conditioned medium from stem cells under oxidative stress (Fig. 5B, C). Interestingly, the strongest band to appear in the gel was at $28 \mathrm{kDa}$, corresponding either to the truncated BDNF form [25] or BDNF-BDNF dimer [26]. Either way, both forms are biologically functional and are capable of binding to the BDNF receptors.

As periodontal ligament cells express surface receptors where BDNF may bind to $[27,28]$, it is plausible to consider that this trophic factor may be an important factor 
A

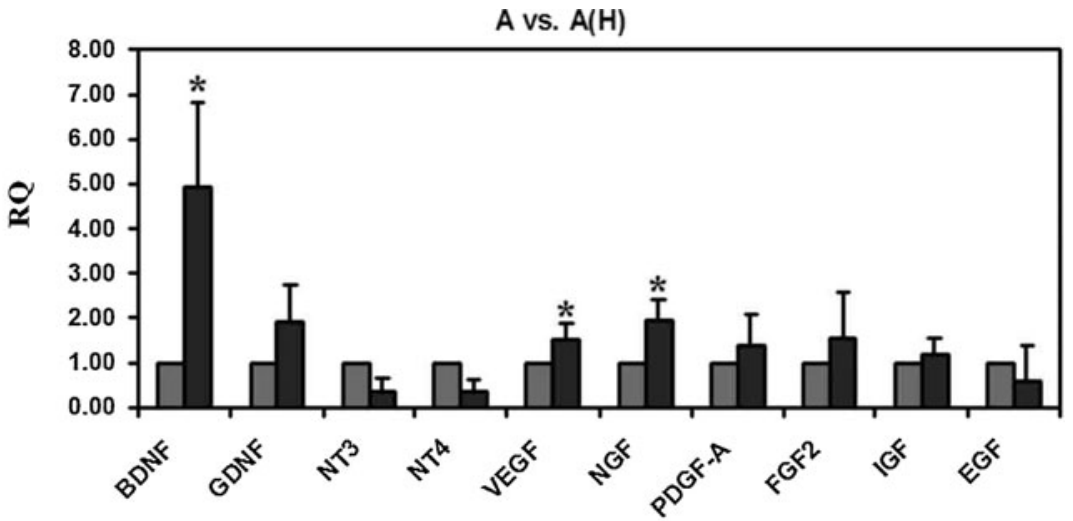

B

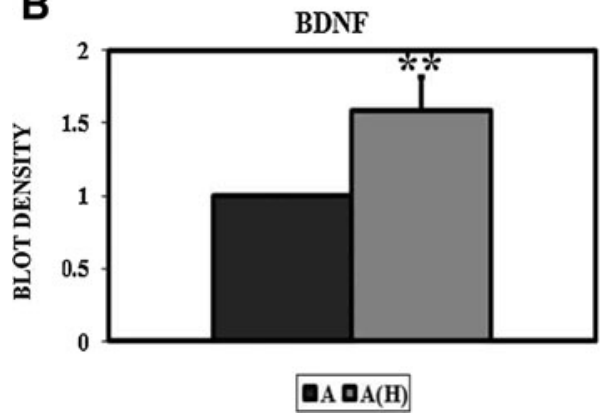

C

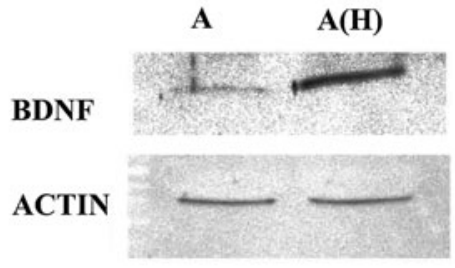

FIG. 5. Trophic factors released by adipose-tissue-derived stem cells. (A) Histogram depicting various trophic factors released by adipose stem cells under normal culture conditions (A, gray bar) compared with the cells cultured in the presence of hydrogen peroxide $[\mathrm{A}(\mathrm{H})$, black bar $]$. Adipose stem cells under normal conditions are considered as control (value $=1$ ). Yaxis indicates the relative quantity of the genes analyzed (RQ). The most significant increases in response to hydrogen peroxide are brain-derived neurotrophic factor (BDNF), nerve growth factor (NGF), and vascular endothelial growth factor (VEGF). All genes are expressed relative to GAPDH. Error bars indicate average deviations: ${ }^{*} P<0.05$. (B, C) Western blot analysis of BDNF in conditioned medium from adipose stem cells under standard culture medium (black bar) and exposed to hydrogen peroxide (gray bar). ${ }^{* *} P<0.01$ ( $n=6$ in all cases). responsible for the increase in cell survival observed, as well as the upregulation of the genes analyzed.

\section{BDNF increases the expression of SODs, NOXs, frataxin, and iron-metabolism-related molecules in response to hydrogen peroxide}

To confirm whether BDNF alone is capable of protecting FA periodontal ligament cells from oxidative stress, we cocultured for 2 days the cells in different concentrations of the trophic factor $(1-100 \mathrm{ng} / \mathrm{mL})$. There was a dose-dependent response to BDNF, being the most significant improvement when the trophic factor was used at $100 \mathrm{ng} / \mathrm{mL}$. Active caspase- 3 was analyzed by quantitative PCR, resulting in less expression of this gene in cells cultured with BDNF (Fig. 6A). As in the other culture conditions, oxidative stress and ironmetabolism-related genes were analyzed, several of these being significantly upregulated. These results were similar to those seen with the adipose stem cell-conditioned medium (see Fig. 4B). Thus, BDNF seems to be capable of protecting FA cells from oxidative stress and may be at least partially responsible for the increase in cell survival, as well as upregulation of the genes analyzed.

To confirm the protective effect of BDNF under oxidative stress, the receptor for BDNF, TrkB, was blocked using a recombinant human antibody. Periodontal ligament cells cultured with this antibody were not affected (Fig. 6C). However, when the periodontal ligament cells were cultured with the antibody and exposed to hydrogen peroxide, there was massive cell death, even under stem-cell-conditioned medium (Fig. 6D-F, respectively). This was clearly visible in the cultures, where numerous cells died and detached from the culture dishes. Active caspase- 3 and Hoechst staining was performed and quantified in the various cultures. As a result, $2.75 \% \pm 0.47 \%$ of the periodontal ligament cells under normal cell culture and with the TrkB blocker presented pyknotic nuclei, and $2.60 \% \pm 1.48 \%$ were active caspase- 3 positive. In the other culture conditions, not only was there a very significant loss of cell density, but also significantly higher percentages of pyknotic nuclei $(7.73 \% \pm 1.16 \%$ in the case of periodontal ligament cells exposed to hydrogen peroxide, $10.32 \% \pm 4.23 \%$ in the case of periodontal ligament cells exposed to hydrogen peroxide and under stem-cellconditioned medium, and $10.31 \% \pm 1.87 \%$ in the cells exposed to hydrogen peroxide and under conditioned medium from adipose stem cells that have previously been submitted to oxidative stress, $n=3$ ). This was corroborated by active caspase- 3 immunostaining $(2.99 \% \pm 0.53 \%$ of the periodontal ligament cells exposed to hydrogen peroxide, $1.35 \% \pm 0.90 \%$ of the cells under stem-cell-conditioned medium, and $4.65 \% \pm 3.10 \%$ of the cells under conditioned medium from stem cells that had previously been submitted to oxidative stress, $n=3)$.

Active caspase- 3 gene expression was also analyzed by quantitative PCR (Fig. 6G, left histogram). As a result, all treatments presented higher active caspase-3 expression compared with control cultures, coinciding with the immunohistochemical analysis. Further, the gene expression analysis by quantitative PCR indicated no significant difference in the expression of frataxin, SOD1, SOD2, catalase, NOX3, DUOX2, or L-ferritin. However, the expression levels of the other genes (SOD3, GPX-1, NOX2, NOX4, NOX5, DUOX1, transferrin, and $H$-ferritin) were increased in the periodontal ligament cells exposed to hydrogen peroxide and under conditioned medium of stem cells submitted to oxidative stress, despite the TrkB blocker. Thus, blocking the BDNF receptor decreased the effectiveness of the adipose stem cellconditioned medium, increasing cell death, although other factors in the conditioned medium were capable of upregulating oxidative-stress-related genes. 

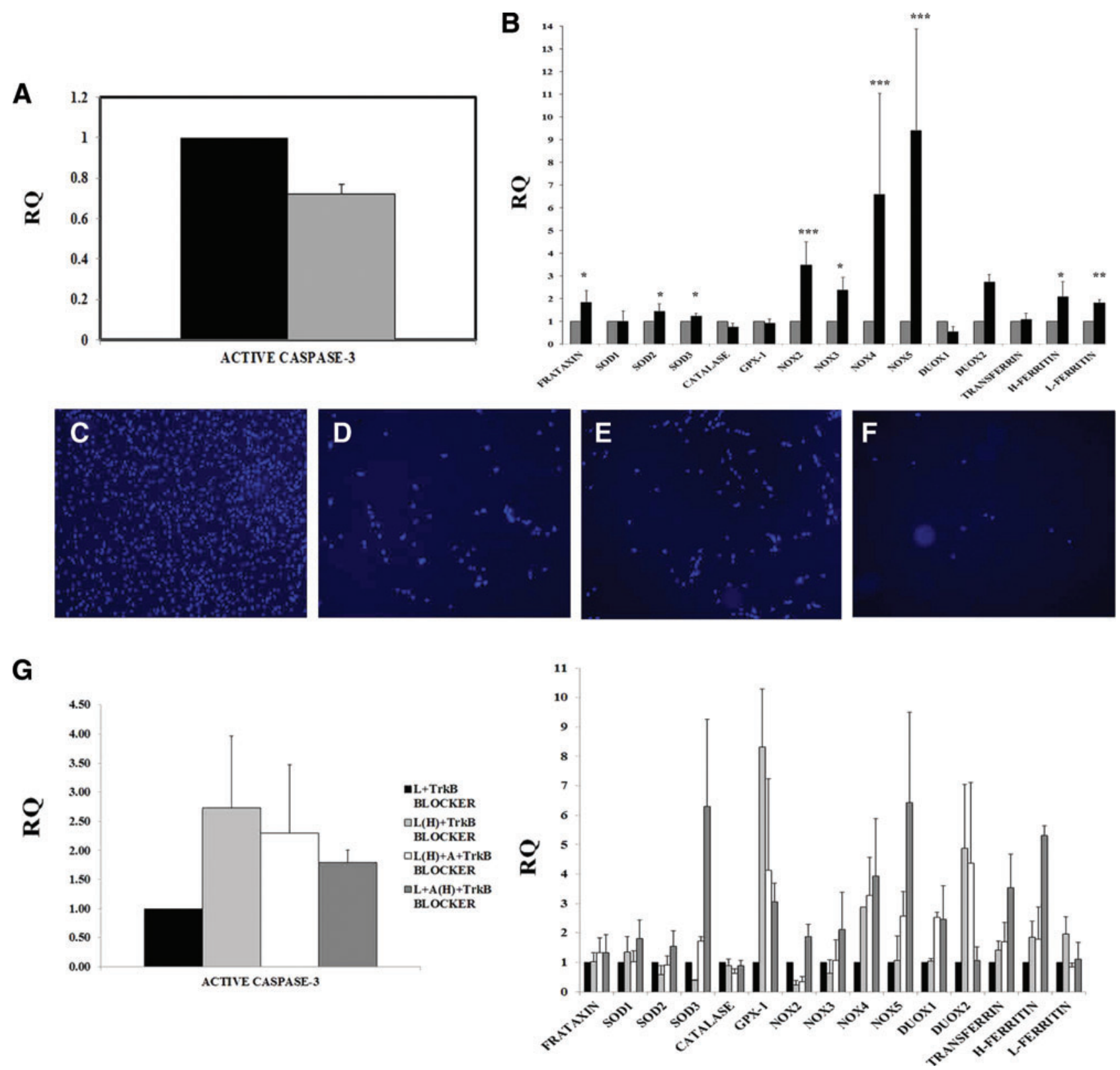

FIG. 6. Gene expression analysis of periodontal ligament cells cultured in $100 \mathrm{ng} / \mathrm{mL}$ BDNF, and with recombinant TrkB antibody. (A) Active caspase-3 expression as measured by QPCR periodontal ligament cells exposed to hydrogen peroxide and with or without BDNF (gray and black bars, respectively). (B) Gene expression levels measured by QPCR in periodontal ligament cells exposed to hydrogen peroxide without BDNF (black bars) and with the trophic factor (gray bars). NOX1 was not detected. ${ }^{*} P<0.05,{ }^{* *} P<0.005$, and ${ }^{* * *} P<0.001$ ( $n=4$ in control and experimental groups). (C-F) Periodontal ligament cells cultured with $100 \mathrm{ng} / \mathrm{mL}$ recombinant TrkB antibody. Images were taken after $48 \mathrm{~h}$ of culture. The images correspond to periodontal ligament cells with recombinant antibody and in normal culture medium (C), exposed to hydrogen peroxide (D), exposed to hydrogen peroxide and in adipose stem cell-conditioned medium (E), and exposed to hydrogen peroxide and in conditioned medium from adipose stem cells previously submitted to oxidative stress (F). Blue is Hoechst staining. All images were taken at $40 \times$ magnification. (G) QPCR analysis of the genes depicted on the histogram of the various cell culture conditions in the presence of the recombinant TrkB antibody. Periodontal ligament cells under standard culture medium and with the TrkB blocker are used as control (value =1). In all histograms, Y-axis indicates the relative quantity of the genes analyzed (RQ). $n=4$ in all cases. Color images available online at www.liebertpub.com/scd

\section{Discussion}

The work presented here shows for the first time that adipose-tissue-derived stem cells are capable of protecting FA cells submitted to oxidative stress. This is due to the secretion of trophic factors in response to the damage, mainly
BDNF. In response to this trophic factor, the ataxic cells increase frataxin expression $\sim 4$ times, while increasing the expression of many genes implicated in hydrogen peroxide removal, as well as other factors implicated in iron metabolism (ferritins and transferrin). Recently, an article has been published showing that bone marrow mesenchymal stem cell- 
conditioned medium from healthy patients had similar effects on fibroblasts isolated from FA patients as those observed in this work [29]. Our work elucidates on the possible mechanisms by which the conditioned medium induces the increase in cell survival as well as frataxin upregulation. Particularly, it seems that the release of certain neurotrophic factors seems to be the main cause for the observed effect.

It has been previously shown by other authors that adipose stem cells, similar to bone marrow cells, are capable of expressing and secreting many trophic factors, including BDNF [30] and NGF [31]. On the other hand, periodontal ligament cells present surface receptors where BDNF may bind to, such as LNGFR (low-affinity NGF receptor, also known as p75) [27] and TrkB [28]. This trophic factor is known to be implicated in processes of cell survival, differentiation, and proliferation, among other functions [32,33]. Further, it has been shown that inoculating BDNF transgene into an ataxic mouse model improved the behavior test in ataxia cells, corroborating that this trophic factor is an important factor for neuroprotection in these disorders [34]. In our lab, we saw that bone marrow mesenchymal stem cells are capable of protecting Purkinje cells in a cerebellar ataxia model by expressing BDNF and to a lesser degree NT3 and GDNF, resulting in improved behavior results [19].

Interestingly, our western blot analysis of the conditioned media showed that the most abundant form of BDNF being secreted is a $28 \mathrm{kDa}$ isoform. The mature form of BDNF is $14 \mathrm{kDa}$, while the pro-BDNF (in the endoplasmic reticulum) weighs $32 \mathrm{kDa}$. The $28 \mathrm{kDa}$ may either be a BDNF dimer, which is also secreted and is fully functional [26], or it may be a truncated, immature form of BDNF, which is also biologically active [25]. Either way, this isoform is completely capable of binding to its ligands, mainly TrkB, thus increasing cell survival.

With these results, it would indicate that BDNF administration could help protect the neurons and cardiomyocytes in FA patients. Indeed, BDNF administration was initially performed in ALS patients, but failed since the trophic factor is not capable of surpassing the blood-brain barrier without a proper vehicle [35]. Intrathecal administration of BDNF, on the other hand, seemed a feasible option, but the clinical trials showed no improvement with respect to placebo controls [36-39]. Also, it must be noted that BDNF administration presents a number of secondary effects that may debilitate the patient, such as hypersensitivity to pain [40]. Thus, although it seems that the use of this trophic factor alone may help ameliorate the progression of the disease, previous results in other diseases, such as ALS, indicate the contrary. On the other hand, stem cell transplantation may act as a biological pump that administers not only BDNF but also other trophic factors in the correct dosage without the side effects commented previously.

Thus, our work demonstrates that adipose-tissue-derived stem cells of healthy individuals are capable of protecting FA cells from oxidative stress and induce the expression of genes implicated in iron metabolism, including and most importantly frataxin.

\section{Acknowledgments}

The authors appreciate the help of F. Rodriguez, M. Ródenas, O. Bahamonde, A. Estirado, and A. Torregrosa for their technical assistance. This work has been financed by
EUCOMMTOOLS, Science and Innovation Ministry (MICINN BFU-2008-00588, CONSOLIDER CSD2007-00023), Valencian government (PROMETEO/2009/028), Cell Therapy Network-Carlos III Health Institute (RD06/0010/0023), Alicia Koplowitz Foundation, Granada Foundation of Friedreich's Ataxia, 5P- Syndrome Foundation, and Diógenes Foundation/Elche (CATEDRA ELA).

\section{Author Disclosure Statement}

The authors state that there is no potential conflict of interest.

\section{References}

1. Campuzano V, L Montermini, MD Molto, L Pianese, M Cossee, F Cavalcanti, E Monros, F Rodius, F Duclos, et al. (1996). Friedreich's ataxia: autosomal recessive disease caused by an intronic GAA triplet repeat expansion. Science 271:1423-1427.

2. Campuzano V, L Montermini, Y Lutz, L Cova, C Hindelang, S Jiralerspong, Y Trottier, SJ Kish, B Faucheux, et al. (1997). Frataxin is reduced in Friedreich ataxia patients and is associated with mitochondrial membranes. Hum Mol Genet 6:1771-1780.

3. Pandolfo M. (2009). Friedreich's ataxia: the clinical picture. J. Neurol 256:3-8.

4. Schulz JB, S Boesch, K Burk, A Durr, P Giunti, C Mariotti, F Pousset, L Schols, P Vankan and M Pandolfo. (2009). Diagnosis and treatment of Friedreich ataxia: a European perspective. Nat Rev Neurol 5:222-234.

5. Alvarez-Dolado M, R Pardal, JM Garcia-Verdugo, JR Fike, HO Lee, K Pfeffer, C Lois, SJ Morrison and A Alvarez-Buylla. (2003). Fusion of bone-marrow-derived cells with Purkinje neurons, cardiomyocytes and hepatocytes. Nature 425:968-973.

6. Weimann JM, CA Charlton, TR Brazelton, RC Hackman and HM Blau. (2003). Contribution of transplanted bone marrow cells to Purkinje neurons in human adult brains. Proc Natl Acad Sci U S A 100:2088-2093.

7. Weimann JM, CB Johansson, A Trejo and HM Blau. (2003). Stable reprogrammed heterokaryons form spontaneously in Purkinje neurons after bone marrow transplant. Nat Cell Biol 5:959-966.

8. Johansson CB, S Youssef, K Koleckar, C Holbrook, R Doyonnas, S Corbel, L Steinman, F Rossi and H Blau. (2008). Extensive fusion of haemotopoietic cells with Purkinje neurons in response to chronic inflammation. Nat Cell Biol 10:575-583.

9. Chen Q, Y Long, X Yuan, L Zou, J Sun, S Chen, JR PerezPolo and K Yang. (2005). Protective effects of bone marrow stromal cell transplantation in injured rodent brain: synthesis of neurotrophic factors. J Neurosci Res 80:611-619.

10. Caplan AI and JE Dennis. (2006). Mesenchymal stem cells as trophic mediators. J Cell Biochem 98:1076-1084.

11. Rodrigues-Hell RC, MM Silva-Costa, A Miranda-Goes and ALR Oliveira. (2009). Local injection of BDNF producing mesenchymal stem cells increases neuronal survival and synaptic stability following ventral root avulsion. Neurobiol Dis 33:290-300.

12. Constantin G, S Marconi, B Rossi, S Angiari, L Calderan, E Anghileri, B Gini, SD Bach, M Martinello, et al. (2009). Adipose-derived mesenchymal stem cells ameliorate chronic experimental autoimmune encephalomyelitis. Stem Cells 27:2624-2635.

13. Brazelton TR, FM Rossi, GI Keshet and HM Blau. (2000). From marrow to brain: expression of neuronal phenotypes in adult mice. Science 290:1775-1779. 
14. Bonilla S, A Silva, L Valdés, E Geijo, JM García-Verdugo and S Martínez. (2005). Functional neural stem cells derived from adult bone marrow. Neuroscience 133:85-95.

15. Rivera FJ, S Couillard-Despres, X Pedre, S Ploetz, M Caioni, C Lois, U Bogdahn and L Aigner. (2006). Mesenchymal stem cell instruct oligodendrogenic fate decision on adult neural stem cells. Stem Cells 24:2209-2219.

16. Wang Y, W Tu, Y Lou, A Xie, X Lai, F Guo and Z Deng. (2009). Mesenchymal stem cells regulate the proliferation and differentiation of neural stem cells through Notch signaling. Cell Biol Int 33:1173-1179.

17. Cabanes C, S Bonilla, L Tabares and S Martínez. (2007). Neuroprotective effect of adult hematopoietic stem cells in a mouse model of motoneuron degeneration. Neurobiol Dis 26:408-418.

18. Pastor D, MC Viso-Leon, J Jones, J Jaramillo-Merchán, JJ Toledo-Aral, JM Moraleda and S Martinez. (2011). Comparative effects between bone marrow and mesenchymal stem cell transplantation in gdnf expression and motor function recovery in a motorneuron degenerative mouse model. Stem Cell Rev 8:445-458.

19. Jones J, J Jaramillo-Merchán, C Bueno, D Pastor, MC VisoLeón and S Martínez. (2010). Mesenchymal stem cells rescue Purkinje cells and improve motor functions in a mouse model of cerebellar ataxia. Neurobiol Dis 40:415-423.

20. Kern S, H Eichler, J Stoeve, H Klüter and K Bieback. (2006). Comparative analysis of mesenchymal stem cells from bone marrow, umbilical cord blood, or adipose tissue. Stem Cells 24:1294-1301.

21. Palomo GM, T Cerrato, R Gargini and J Diaz-Nido. (2011). Silencing of frataxin gene expression triggers p53-dependent apoptosis in human neuron-like cells. Hum Mol Genet 20:2807-2822.

22. Huang CY, D Pelaez, J Dominguez-Bendala, F Garcia-Godoy and HS Cheung. (2009). Plasticity of stem cells derived from adult periodontal ligament. Regen Med 4:809-821.

23. Irazusta V, E Obis, A Moreno-Cermeño, E Cabiscol, J Ros and J Tamarit. (2010). Yeast frataxin mutants display decreased superoxide dismutase activity crucial to promote protein oxidative damage. Free Radic Biol Med 48:411-420.

24. Marmolino D, M Manto, F Acquaviva, P Vergara, A Ravella, A Monticelli and M Pandolfo. (2010). PGC-1alpha downregulation affects the antioxidant response in Friedreich's ataxia. PLoS One 5:e10025.

25. Mowla SJ, HF Farhadi, S Pareek, JK Atwal, SJ Morris, NG Seidah and RA Murphy. (2001). Biosynthesis and posttranslational processing of the precursor to brain-derived neurotrophic factor. Biol Chem 276:12660-12666.

26. Philo J, J Talvenheimo, J Wen, R Rosenfeld, A Welcher and T Arakawa. (1994). Interactions of neurotrophin-3 (NT-3), brain-derived neurotrophic factor (BDNF), and the NT3.BDNF heterodimer with the extracellular domains of the TrkB and TrkC receptors. J Biol Chem 269:27840-27846.

27. Coura GS, RC Garcez, CB de Aguiar, M Alvarez-Silva, RS Magini and AG Trentin. (2008). Human periodontal ligament: a niche of neural crest stem cells. J Periodontal Res 43:531-536.

28. Mizuno N, H Shiba, T Inui, K Takeda, M Kajiya, N Hasegawa, H Kawaguchi and H Kurihara. (2008). Effect of neurotrophin-4/5 on bone/cementum-related protein expressions and DNA synthesis in cultures of human periodontal ligament cells. J. Periodont 79:2182-2189.

29. Kemp K, E Mallam, K Hares, J Witherick, N Scolding and A Wilkins. (2011). Mesenchymal stem cells restore frataxin expression and increase hydrogen peroxide scavenging enzymes in Friedreich ataxia fibroblasts. PLOS One 6:e26098.

30. Lopatina T, N Kalinina, M Karagyaur, D Stambolsky, K Rubina, A Revishin, G Pavlova, Y Parfyonova and V Tkachuk. (2011). Adipose-derived stem cells stimulate regeneration of peripheral nerves: BDNF secreted by these cells promotes nerve healing and axon growth de novo. PLOS One 6:e17899.

31. Tan B, Z Luan, X Wei, Y He, G Wei, BH Johnstone, M Farlow and Y Du. (2011). AMP-activated kinase mediates adipose stem cell-stimulated neuritogenesis of PC12 cells. Neuroscience 181:40-47.

32. Schwartz PM, PR Borghesani, RL Levy, SL Pomeroy and RA Segal. (1997). Abnormal cerebellar development and foliation in BDNF - / - mice reveals a role for neurotrophins in CNS patterning. Neuron 19:269-281.

33. Segal RA, H Takahashi and RD McKay. (1992). Changes in neurotrophin responsiveness during the development of cerebellar granule neurons. Neuron 9:1041-1052.

34. Meng H, S Larson, R Gao and X Qiao. (2007). BDNF transgene improve ataxic and motor behaviors in stargazer mice. Brain Res 1160:47-57.

35. Pardridge WM, YS Kang and JL Buciak. (1994). Transport of human recombinant brain-derived neurotrophic factor (BDNF) through the rat blood-brain barrier in vivo using vector-mediated peptide drug delivery. Pharm Res 11: 738-746.

36. Bradley WG, W Hedlund, C Cooper, et al. (1984). A doubleblind controlled trial of bovine brain gangliosides in amyotrophic lateral sclerosis. Neurology 34:1079-1082.

37. The BDNF Study Group (Phase III). (1999). A controlled trial of recombinant methionyl human BDNF in ALS: Neurology 52:1427-1433.

38. Kalra S, A Genge and DL Arnold. (2003). A prospective, randomized, placebo-controlled evaluation of corticoneuronal response to intrathecal BDNF therapy in ALS using magnetic resonance spectroscopy: feasibility and results. Amyotroph Lateral Scler Other Motor Neuron Disord 4: 22-26.

39. Beck M, P Flachenecker, T Magnus, R Giess, K Reiners, KV Toyka and M Naumann. (2005). Autonomic dysfunction in ALS: a preliminary study on the effects of intrathecal BDNF. Amyotroph Lateral Scler Other Motor Neuron Disord 6: 100-103.

40. Li CQ, JM Xu, D Liu, JY Zhang and RP Dai. (2008). Brain derived neurotrophic factor (BDNF) contributes to the pain hypersensitivity following surgical incision in the rats. Mol Pain 4:27-37.

Address correspondence to: Dr. Jonathan Jones Neuroscience Institute UMH-CSIC University Miguel Hernández Crtra. Valencia km. 87 San Juan, Alicante, 03550 Spain E-mail: jon@umh.es

Received for publication January 23, 2012 Accepted after revision May 1, 2012 Prepublished on Liebert Instant Online May 1, 2012 